ANNALES

UNIVERSITATIS MARIAE C URIE-SKŁODOW K A LUBLIN - POLONIA

VOL. LXXI, 2
SECTIO C

2016

\author{
ANETA CZARNA \\ Department of Botany \\ Poznań University of Life Sciences \\ ul. Wojska Polskiego 71c, 60-625 Poznań, Poland \\ e-mail: czarna@up.poznan.pl

\section{Vascular plants in the Cemetery of the Meritorious in Poznań (Poland)}

\begin{abstract}
SUMMARY
The Cemetery of the Meritorious is the oldest preserved burial site in the city of Poznan (western Poland). It was created in 1808, but after World War II it was subject to gradual devastation. In 1961, it was classified as a historic site, completely protected by law and cared for by the Cultural Heritage Officer. Field research shows that the vascular flora of the cemetery is composed of 140 taxa: 15 in the trees, 26 in the shrubs, and 99 in the herbaceous plants. The flora comprises 84 taxa $(57.1 \%)$ that were planted there. Most of them are herbaceous: 45 species $(30.6 \%)$, including 14 native $(9.5 \%)$ and 31 alien ones (21.1\%). Plants with symbolic meanings are represented by 13 taxa in the trees, 16 in the shrubs, and 30 in the herbaceous plants. Currently, however, the symbolism of cemetery plants is of little significance, as they are cultivated primarily because of their ornamental value. Special attention was paid to groundcover plants, represented by 37 species $(25,2 \%)$, including 21 taxa introduced a long time ago and 16 recently (during the last 10 years or so).
\end{abstract}

Keywords: vascular flora, groundcover plants, Cemetery of the Meritorious, St. Adalbert's Hill, historic site, Poznań, Wielkopolska, Poland

\title{
STRESZCZENIE
}

Cmentarz Zasłużonych Wielkopolan jest najstarszym zachowanym miejscem pochówku w Poznaniu. Został utworzony w 1808 r. W latach powojennych uległ stopniowej dewastacji. W 1961 r. uznano go za obiekt zabytkowy, w całości podległy opiece konserwatora. Na omawianym cmentarzu stwierdzono 140 gatunków roślin naczyniowych. Wśród drzew wystąpiło 15 gatunków, krzewów 26, a wśród roślin zielnych 99. Wśród całej flory naczyniowej jest 84 taksonów (57,5\%) wprowadzonych do uprawy. Najwięcej jest ich w warstwie zielnej: 45 gatunków 
(30,8\%), wśród których $14(9,6 \%)$ są to gatunki rodzime i $31(21,2 \%)$ to gatunki obce. Wśród roślin o znaczeniu symbolicznym jest 13 drzew, 16 krzewów i 30 roślin zielnych. Obecnie symbolika związana z roślinnością cmentarną odgrywa niewielką rolę, a rośliny sadzone są głównie ze względu na walory dekoracyjne. Na szczególne podkreślenie zasługują gatunki okrywowe, wśród których stwierdzono 37 gatunków (24,6\%), z czego 21 taksonów wprowadzono w dawnych czasach, a 16 współcześnie (w ciągu 10 lat).

Słowa kluczowe: flora naczyniowa, gatunki okrywowe, Cmentarz Zasłużonych, Wzgórze św. Wojciecha, obiekt zabytkowy, Poznań,Wielkopolska, Polska

\section{INTRODUCTION}

Thanatourism, i.e. travelling to places historically associated with death, such as cemeteries, is a more and more popular form of cultural tourism. Attention is paid to tombstones and monuments, but also to the introduced ornamental plants, which add a lot of charm and colour to old cemeteries. Thanatourism includes also visits to small abandoned cemeteries in villages, where cemetery phytoindicators (indicator plants) and noteworthy symbolic plants have survived. Thanatouristic trails can be designated in many regions, also in the city of Poznań.

Municipal cemeteries are some of the most valuable urban green areas, where trees play an important role. The selection of herbaceous plants is strongly affected by environmental conditions, e.g. light, water, microclimate, and soil type. It is recommended that in cemeteries low plants should be preferred, with small root systems, resistant to drought (9). In the past, necropoles were dependent on religious beliefs and were major sites in the city centre. In the course of time, various meanings were attributed to cemetery plants, usually linked with paradise imagery (17).

This study was aimed to analyse the taxonomic composition of vascular plants in the Cemetery of the Meritorious in Poznań, with particular reference to symbolic plants. It was tested whether a cemetery subject to continuous care is characterized by a richness of groundcover plants introduced to cultivation under trees and by a small contribution of apophytes (i.e. native plants found on sites disturbed by human activity).

\section{STUDY SITE}

The Cemetery of the Meritorious is located in the city of Poznan in western Poland, on St. Adalbert's Hill (Wzgórze św. Wojciecha), near St. Joseph's Church and a cloister of Barefoot Carmelites. It is the oldest necropolis in Poznań, as on 19 February 1810, the first burial took place on the new, then still disorganized cemetery. It is exceptionally picturesque, situated in an area with varied relief: a wooded slope of the hill, with north-eastern exposure, and a basin-like, slightly concave glade, which is a remnant of a former floodplain of a tributary of the stream Wierzbak. Part of the cemetery, with no graves and monuments, is a burial site of numerous victims of the cholera epidemics that swept Poznań in 1831-1873 (12).

The cemetery on St. Adalbert's Hill is also a burial site of many citizens of Poznan and of the Wielkopolska region who died in fights for Polish independence, but also of those who devoted their lives to helping others with their hard work. In 1948, the cemetery started to be supervised by the Municipal Council and since then only the most meritorious citizens of the Wielkopolska region can be buried there. After World War II, the cemetery was subject to gradual devastation until 1961, when it was classified as a historic site, completely protected by law and cared for by the Cultural Heritage Officer. In the 1960s, it was renovated: new lanes and paths were marked out, graves were cleared, while in the park-like part, several hundred rose bushes and other ornamental species were 
planted. In 1980, a social action took place, which resulted not only in renovation of the tombstones and clearance of rubbish, etc., but also initiation of continuous supervision of the cemetery, with a phone connection (12). Currently, new ornamental plants are still planted there and the monuments are being renovated.

\section{MATERIALS AND METHODS}

Field research on the vascular flora of the cemetery was conducted in 2011-2012. Herbarium specimens have been deposited at the Faculty of Forestry, Poznań University of Life Sciences (POZNB). Names of species follow $(6,7,15)$. Frequency of each species was assessed on a 7-point scale: $\mathrm{R}$ - negligible (1-2 plants); + - several plants; 1 - very rare (covering $1-5 \%$ of cemetery area excluding graves); 2 - rare (5-25\%); 3 - moderately frequent $(25-50 \%) ; 4$ - frequent $(50-75 \%)$; and 5 -common (75-100\%). Abbreviation "cul" indicates that the species was planted (long ago or recently, i.e. during the last 10 years or so).

Groundcover plants were classified as such on the basis of three sources $(2,5,13)$. Raunkiaer's life-forms were presented primarily according to $(6)$, but also $(18,19)$. The socioecological classification was based mostly on (12). Protected species were determined on the basis of the Regulation of the Minister of Environmental Protection of 16 October 2014, while plants with symbolic meanings, by $(10,11)$ and $(20)$.

\section{RESULTS}

During the research in the Cemetery of the Meritorious on St. Adalbert's Hill in Poznań, 140 vascular plant taxa were recorded (Tab. 1). Some species were found simultaneously in the tree, shrub, and herb layers (Hedera helix) or in both tree and shrub layers (Robinia pseudoacacia) or in both shrub and herb layers (Taxus baccata) or in both tree and herb layers (Acer platanoides, Aesculus hippocastanum, Ailanthus altissima, and Tilia cordata). Among the recorded species, as many as 81 taxa (55.1\%) were planted there.

Table 1. Vascular flora in the Cemetery of the Meritorious in Poznań: frequency classes (FC), life-forms (LF), geographic-historical status (GH), socioecological groups (SE), and symbolic meaning (Symb.)

\begin{tabular}{|c|c|c|c|c|c|}
\hline Species & $\mathrm{FC}$ & $\mathrm{LF}$ & GH & SE & Symb. \\
\hline \multicolumn{6}{|l|}{ Tree layer } \\
\hline Abies concolor (Gordon \& Glend.) Lindl. Ex Hildebr. & 1 culA & F1 & Er1 & 17 & $\mathrm{~S}$ \\
\hline Acer platanoides $\mathrm{L}$. & 3 culA & F1 & Ap2 & 1 & $\mathrm{~S}$ \\
\hline Acer pseudoplatanus L. & 1 culA & F1 & Ap2 & 1 & $\mathrm{~S}$ \\
\hline Aesculus hippocastanum L. & 2 culA & F1 & $\mathrm{Kn}$ & 17 & $\mathrm{~S}$ \\
\hline Ailanthus altissima (Mill.) Swingle & 1 culA & F1 & $\mathrm{Kn}$ & 17 & \\
\hline Fraxinus excelsior $\mathrm{L}$. & 2 culA & F1 & Ap2 & 1 & $\mathrm{~S}$ \\
\hline Fraxinus excelsior L. 'Pendula' & 1 culA & F1 & Ap2 & 1 & $\mathrm{~S}$ \\
\hline Hedera helix $\mathrm{L}$ & 1 culA & C1 li & $\mathrm{Kn}$ & 1 & $\mathrm{~S}$ \\
\hline
\end{tabular}


Pobrane z czasopisma Annales C - Biologia http://biologia.annales.umes.pl

Data: 26/04/2023 14:01:33

62

ANETA CZARNA

Tab. 1

\begin{tabular}{|c|c|c|c|c|c|}
\hline Species & $\mathrm{FC}$ & $\mathrm{LF}$ & GH & $\mathrm{SE}$ & Symb. \\
\hline Pseudotsuga menziesii (Mirb.) Franco & 1 culA & $\mathrm{F} 1$ & $\mathrm{Kn}$ & 2 & \\
\hline Quercus robur L. & 2 culA & $\mathrm{F} 1$ & Ap2 & 1 & $\mathrm{~S}$ \\
\hline Robinia pseudoacacia L. & $1 \mathrm{culA}$ & F1 & $\mathrm{Kn}$ & 12 & $\mathrm{~S}$ \\
\hline Tilia cordata Mill. & 2 culA & F1 & Ap2 & 1 & $\mathrm{~S}$ \\
\hline Tilia 'Euchlora' & 1 culA & $\mathrm{F} 1$ & Er1 & 17 & $\mathrm{~S}$ \\
\hline Tilia platyphyllos Scop. & 1 culA & F1 & Ap2 & 1 & $\mathrm{~S}$ \\
\hline Viscum album $\mathrm{L}$. & + & C2ppep & $\mathrm{Kn}$ & 6 & $\mathrm{~S}$ \\
\hline \multicolumn{6}{|l|}{ Shrub layer } \\
\hline Acer tataricum $\mathrm{L}$. & + culB & $\mathrm{F} 1$ & Er1 & 17 & \\
\hline Buxus sempervirens L. & + culA & F2 & Er1 & 17 & $\mathrm{~S}$ \\
\hline Carpinus betulus L. & + culB & $\mathrm{F} 2$ & Ap2 & 1 & \\
\hline Chamaecyparis pisifera (Siebold\& Zucc.) Endl.. & $1 \mathrm{culB}$ & $\mathrm{F} 1$ & Er1 & 17 & $\mathrm{~S}$ \\
\hline Cornus stolonifera Michx. & $1 \mathrm{culB}$ & $\mathrm{F} 2$ & Er1 & 17 & \\
\hline $\begin{array}{l}\times \text { Cupressocyparis leylandii (A.B.Jacks. et } \\
\text { Dallim.) Dallim. }\end{array}$ & + culB & $\mathrm{F} 1$ & Er1 & 17 & $\mathrm{~S}$ \\
\hline Deutzia gracilis Siebold \& Zucc. & + culB & $\mathrm{F} 2$ & Er1 & 17 & \\
\hline Forsythia $\times$ intermedia Zabel & $1 \mathrm{culB}$ & $\mathrm{F} 2$ & Er1 & 17 & \\
\hline Fraxinus pennsylvanica Marshall & + & F1 & $\mathrm{Kn}$ & 17 & $\mathrm{~S}$ \\
\hline Hedera helix $\mathrm{L}$. & $+\operatorname{culA}$ & C1 li & $\mathrm{Kn}$ & 1 & $\mathrm{~S}$ \\
\hline Hamamelis mollis Oliv. & $+\mathrm{culB}$ & $\mathrm{F} 2$ & Er1 & 17 & \\
\hline Juniperus virginiana L. & + culA & $\mathrm{F} 1$ & Er1 & 17 & $\mathrm{~S}$ \\
\hline Kerria japonica (L.) DC. & + culB & $\mathrm{F} 2$ & Er1 & 17 & \\
\hline Mahonia aquifolium (Pursh) Nutt. & + culA & $\mathrm{F} 2$ & Er1 & 17 & $\mathrm{~S}$ \\
\hline Philadelphus coronarius L. & $+\operatorname{culA}$ & $\mathrm{F} 2$ & Er1 & 17 & \\
\hline Physocarpus opulifolius (L.) Maxim. & + culB & $\mathrm{F} 2$ & Er1 & 17 & \\
\hline Picea pungens Engelm. & $+\operatorname{culA}$ & $\mathrm{F} 2$ & Er1 & 17 & $\mathrm{~S}$ \\
\hline Robinia pseudoacacia L. & + & F1 & $\mathrm{Kn}$ & 12 & $\mathrm{~S}$ \\
\hline Rosa chinensis Jacq. & + culB & $\mathrm{F} 2$ & Er1 & 17 & $\mathrm{~S}$ \\
\hline Sambucus nigra L. & + & $\mathrm{F} 2$ & Ap1 & 3 & $\mathrm{~S}$ \\
\hline Spiraea japonica L. f. & + culB & $\mathrm{F} 2$ & Er1 & 17 & \\
\hline Symphoricarpos albus (L.) S. F. Blanke & 1 culA & $\mathrm{F} 2$ & Er1 & 17 & \\
\hline Symphoricarpos ×chenaultii Rehd. & + culB & $\mathrm{F} 2$ & Er1 & 17 & \\
\hline Syringa $\times$ chinensis Willd. & $+\operatorname{culA}$ & $\mathrm{F} 2$ & Er1 & 17 & $\mathrm{~S}$ \\
\hline Syringa vulgaris L. & + culA & $\mathrm{F} 2$ & Er1 & 17 & $\mathrm{~S}$ \\
\hline Taxus baccata $\mathrm{L}$. & 1 culA & $\mathrm{F} 2$ & Ap2 & 1 & $\mathrm{~S}, \mathrm{Ccz}$ \\
\hline Thuja occidentalis L. & $+\operatorname{culA}$ & F1 & Er1 & 17 & $\mathrm{~S}$ \\
\hline Tsuga canadensis (L.) Carričre & + culB & F1 & Er1 & 17 & \\
\hline Viburnum rhytidophyllum Hemsl. & + culB & $\mathrm{F} 2$ & Er1 & 17 & $\mathrm{~S}$ \\
\hline
\end{tabular}


Pobrane z czasopisma Annales C - Biologia http://biologia.annales.umcs.pl

Data: 26/04/2023 14:01:33

VASCULAR PLANTS IN THE CEMETERY OF THE MERITORIOUS IN POZNAŃ (POLAND)

63

Tab. 1

\begin{tabular}{|c|c|c|c|c|c|}
\hline Species & $\mathrm{FC}$ & $\mathrm{LF}$ & $\mathrm{GH}$ & SE & Symb. \\
\hline \multicolumn{6}{|l|}{ Herb layer } \\
\hline Acer platanoides $\mathrm{L}$. & 1 & F1 & Ap2 & 1 & $\mathrm{~S}$ \\
\hline Aegopodium podagraria $\mathrm{L}$. & 1 culA & H1 & Ap2 & 1 & \\
\hline Aesculus hippocastanum L. & + & F1 & $\mathrm{Kn}$ & 17 & $\mathrm{~S}$ \\
\hline Alchemilla mollis (Buser) Rothm. & + culB & H1 & Er1 & 17 & $\mathrm{~S}$ \\
\hline Ailanthus altissima (Mill.) Swingle & $\mathrm{R}$ & F1 & $\mathrm{Kn}$ & 17 & \\
\hline Alliaria petiolata (M. Bieb) Cavara \& Grande & $\mathrm{R}$ & $\mathrm{T} 1$ & Ap1 & 3 & \\
\hline Allium scorodoprasum L. & 1 culA & G1 & $\mathrm{Kn}$ & 4 & $\mathrm{~S}$ \\
\hline Allium vineale $\mathrm{L}$. & + & G1 & Ap1 & 4 & $\mathrm{~S}$ \\
\hline Anemone ranunculoides L. & 1 culA & G1 & Sp1 & 1 & $\mathrm{~S}$ \\
\hline Anthriscus sylvestris (L.) Hoffm. & + & $\mathrm{H} 1$ & Ap1 & 3 & \\
\hline Aquilegia vulgaris $\mathrm{L}$. & $\mathrm{R}$ culA & $\mathrm{H} 1$ & Sp1 & 3 & $\mathrm{~S}, \mathrm{Ccz}$ \\
\hline Bellis perennis $\mathrm{L}$. & 1 & $\mathrm{H} 1$ & Ap1 & 8 & $\mathrm{~S}$ \\
\hline Bergenia cordifolia (Haw.) Sternb. & + culA & $\mathrm{H} 1$ & Er1 & 17 & \\
\hline Bromus hordaceus L. & + & $\mathrm{T} 1$ & Ap1 & 12 & \\
\hline Campanula rapunculoides $\mathrm{L}$. & $+\operatorname{culA}$ & $\mathrm{H} 1$ & Ap2 & 14 & \\
\hline Capsella bursa-pastoris (L.) Medik. & $\mathrm{R}$ & $\mathrm{T} 1$ & Ach & 14 & \\
\hline Cardamine hirsuta $\mathrm{L}$. & + & $\mathrm{T} 1$ & Ap2 & 14 & \\
\hline Cerastium glomeratum Thuill. & $\mathrm{R}$ & $\mathrm{T} 1$ & Ap1 & 17 & \\
\hline Cerastium holosteoides Fr. Emend. Hyl. & + & H1 & Ap1 & 8 & \\
\hline Cerastium semidecandrum $\mathrm{L}$. & + & $\mathrm{T} 1$ & Ap1 & 4 & \\
\hline Chelidonium majus L. & + & $\mathrm{H} 1$ & Ap1 & 3 & $\mathrm{~S}$ \\
\hline Convallaria majalis $\mathrm{L}$. & $1 \mathrm{culA}$ & G1 & $\mathrm{Sp} 2$ & 3 & $\mathrm{~S}$ \\
\hline Conyza canadensis (L.) Cronquist & + & $\mathrm{T} 1$ & $\mathrm{Kn}$ & 13 & \\
\hline Cotoneaster horizontalis Dence. & $+\operatorname{culA}$ & F2 & Er1 & 17 & \\
\hline Cotoneaster suecicus G. Klotz & 1 culA & F2 & Er1 & 17 & \\
\hline Crocus vernus (L.) Hill & $\mathrm{r}$ culB & G1 & Er1 & 3 & $\mathrm{~S}$ \\
\hline Dactylis glomerata L. & + & $\mathrm{H} 1$ & Ap1 & 8 & \\
\hline Digitaria sanguinalis (L.) Scop. & + & $\mathrm{T} 1$ & $\mathrm{Ar}$ & 11 & \\
\hline Dryopteris filix-mas (L.) Schott & 2 culB & $\mathrm{H} 1$ & Ap1 & 2 & \\
\hline Echinochloa crus-galli (L.) P.Beauv. & + & $\mathrm{T} 1$ & $\mathrm{Ar}$ & 14 & \\
\hline Eranthis hyemalis (L.) Salisb. & + culB & G1 & Er1 & 17 & \\
\hline Erophila verna (L.) Chevell. & 1 & $\mathrm{~T} 1$ & Ap1 & 5 & \\
\hline Euphorbia helioscopia L. & + & $\mathrm{T} 1$ & $\mathrm{Ar}$ & 14 & \\
\hline Fallopia convolvulus (L.) Á. Löve & + & $\mathrm{T} 1$ & $\mathrm{Ar}$ & 14 & \\
\hline Ficaria verna Huds. & 2 culA & G1 & Ap2 & 14 & \\
\hline Fragaria indica Andr. & + culB & $\mathrm{H} 1$ & Er1 & 17 & $\mathrm{~S}$ \\
\hline Gagea arvensis (Pers.) Dumort. & $+\operatorname{culA}$ & G1 & $\mathrm{Ar}$ & 3 & \\
\hline
\end{tabular}


Pobrane z czasopisma Annales C - Biologia http://biologia.annales.umcs.pl

Data: 26/04/2023 14:01:33

64

ANETA CZARNA

Tab. 1

\begin{tabular}{|c|c|c|c|c|c|}
\hline Species & $\mathrm{FC}$ & $\mathrm{LF}$ & $\mathrm{GH}$ & SE & Symb. \\
\hline Gagea lutea (L.) Ker Gawl. & 2 culA & G1 & Ap2 & 1 & \\
\hline Gagea pratensis (Pers.) Dumort. & 1 & G1 & Ap2 & 3 & \\
\hline Galanthus nivalis $\mathrm{L}$. & $+\operatorname{culA}$ & G1 & Ap1 & 1 & $\mathrm{~S}, \mathrm{CCz}$ \\
\hline Galeobdolon argentatum Snejkal & 1 culA & H1 & Er1 & 17 & \\
\hline Galium mollugo L. s.s. & + & H1 & Ap1 & 8 & \\
\hline Geranium macrorrhizum L. & 1 culB & $\mathrm{H} 1$ & Er1 & 17 & \\
\hline Geranium platypetalum Fisch. \& C. A. Mey & + culB & H1 & Er1 & 17 & \\
\hline Geranium molle L. & + & H1 & $\mathrm{Kn}$ & 12 & \\
\hline Geranium pratense L. & + & H1 & Ap1 & 8 & \\
\hline Geranium pusillum Burm. F. ex L. & + & $\mathrm{T} 1$ & $\mathrm{Ar}$ & 14 & \\
\hline Geum urbanum L. & $\mathrm{R}$ & H1 & Ap1 & 3 & $\mathrm{~S}$ \\
\hline Glechoma hederacea L. & + & H1 & Ap1 & 1 & \\
\hline Hedera helix L. & 3 culA & C1 li & $\mathrm{Kn}$ & 1 & $\mathrm{~S}$ \\
\hline Hemerocallis fulva $\mathrm{L}$. & 1 culA & H1 & Er1 & 17 & \\
\hline Heracleum sibiricum L. & + & H1 & Ap1 & 7 & \\
\hline Hyacinthus orientalis L. & RculB & G1 & Er1 & 17 & \\
\hline Lactuca serriola $\mathrm{L}$. & $\mathrm{R}$ & $\mathrm{T} 2$ & $\mathrm{Ar}$ & 15 & \\
\hline Lamium purpureum $\mathrm{L}$. & + & $\mathrm{T} 1$ & $\mathrm{Ar}$ & 14 & \\
\hline Leontodon autumnalis L. & $\mathrm{R}$ & H1 & Ap1 & 9 & \\
\hline Ligularia dentata (A. Gray) H. Hara & + culB & H1 & Er1 & 17 & \\
\hline Lolium perenne L. & 2 & $\mathrm{H} 1$ & Ap1 & 9 & \\
\hline Lysimachia nummularia L. & $+\operatorname{culA}$ & $\mathrm{H} 1$ & Ap2 & 1 & \\
\hline Matteucia struthiopteris (L.) Tod. & $1 \mathrm{culA}$ & H1 & Er1 & 17 & $\mathrm{CCz}$ \\
\hline Muscari botryoides (L.) Mill. & $\mathrm{R}$ culA & G1 & Er1 & 17 & \\
\hline Myosotis sparsiflora Pohl & + & H1 & Ap1 & 6 & $\mathrm{~S}$ \\
\hline Ornithogalum nutans L. & $+\operatorname{culA}$ & G1 & Er2 & 17 & \\
\hline Ornithogalum umbellatum L. & $2 \operatorname{culA}$ & G1 & $\mathrm{Kn}$ & 17 & \\
\hline Oxalis dillenii Jacq. & + & $\mathrm{T} 1$ & $\mathrm{Kn}$ & 15 & \\
\hline Oxalis fontana Bunge & + & $\mathrm{T} 1$ & $\mathrm{Kn}$ & 13 & \\
\hline Pachysandra terminalis Siebold \& Zucc. & $1 \mathrm{culB}$ & $\mathrm{C} 1$ & Er1 & 17 & \\
\hline Paeonia officinalis $\mathrm{L}$. & $\mathrm{R}$ culA & $\mathrm{H} 1$ & Er1 & 17 & $\mathrm{~S}$ \\
\hline Picris hieraciodes L. & $\mathrm{R}$ & H1 & Ap1 & 12 & \\
\hline Pоа аппиа $\mathrm{L}$. & + & $\mathrm{T} 1$ & Ap1 & 9 & \\
\hline Poa nemoralis L. & 1 & $\mathrm{H} 1$ & Ap1 & 2 & \\
\hline Poa pratensis L. s.s. & 2 & H1 & Ap1 & 8 & \\
\hline Polygonum aviculare L. & + & $\mathrm{T} 1$ & Ap1 & 9 & \\
\hline Potentilla reptans $\mathrm{L}$. & $\mathrm{R}$ & H1 & Ap1 & 9 & \\
\hline Prunella vulgaris $\mathrm{L}$. & 1 & H1 & Ap1 & 8 & \\
\hline
\end{tabular}


Pobrane z czasopisma Annales C - Biologia http://biologia.annales.umcs.pl

Data: 26/04/2023 14:01:33

VASCULAR PLANTS IN THE CEMETERY OF THE MERITORIOUS IN POZNAŃ (POLAND)

65

Tab. 1

\begin{tabular}{|c|c|c|c|c|c|}
\hline Species & FC & LF & GH & SE & Symb. \\
\hline Ranunculus bulbosus L. & $\mathrm{R}$ & H1 & Ap1 & 3 & \\
\hline Reynoutria japonica Houtt & $+\operatorname{culA}$ & G1 & Er1 & 17 & \\
\hline Rumex obtusifolius L. & $\mathrm{R}$ & H1 & Apl & 11 & \\
\hline Rumex thyrsiflorus Fingerh. & + & H1 & Ap1 & 12 & \\
\hline Sagina procumbens L. & + & H1 & Ap1 & 10 & \\
\hline Scilla sibirica Haw. & 2 culA & G1 & Er2 & 17 & \\
\hline Sedum spectabile Boreau & 1 culA & $\mathrm{H} 1$ & Er1 & 17 & \\
\hline Senecio vulgaris L. & $\mathrm{R}$ & $\mathrm{T} 1$ & $\mathrm{Ar}$ & 14 & \\
\hline Setaria viridis (L.) P. Beauv. & + & $\mathrm{T} 1$ & $\mathrm{Ar}$ & 14 & \\
\hline Sonchus oleraceus L. & $\mathrm{R}$ & $\mathrm{T} 1$ & $\mathrm{Ar}$ & 14 & \\
\hline Spergularia rubra (L.) J. Presl \& C. Presl & + & $\mathrm{T} 1$ & Ap1 & 5 & \\
\hline Stellaria media (L.) Vill. & + & $\mathrm{T} 1$ & Ap1 & 14 & \\
\hline Stellaria pallida (Dumort.) Piré & + & $\mathrm{T} 2$ & Ap1 & 14 & \\
\hline Taraxacum officinale Web. & 2 & $\mathrm{H} 1$ & Ap1 & 8 & $\mathrm{~S}$ \\
\hline Taxus baccata L. & 1 culA & F2 & Ap2 & 17 & $\mathrm{~S}, \mathrm{CCz}$ \\
\hline Tilia cordata Mill. & + & F1 & Ap1 & 4 & $\mathrm{~S}$ \\
\hline Tradescantia $\times$ andersoniana W. Ludw. \& Rohweder & + culB & H1 & Er1 & 17 & \\
\hline Trifolium pratense $\mathrm{L}$. & + & H1 & Ap1 & 8 & $\mathrm{~S}$ \\
\hline Tulipa gesneriana L. & $\mathrm{r}$ culA & G1 & Er1 & 17 & $\mathrm{~S}$ \\
\hline Tulipa sylvestris L. & 2 culA & G1 & Er2 & 17 & $\mathrm{~S}$ \\
\hline Urtica dioica $\mathrm{L}$. & $\mathrm{R}$ & H1 & Ap1 & 3 & $S$ \\
\hline Veronica arvensis L. & + & $\mathrm{T} 1$ & Ap1 & 15 & $\mathrm{~S}$ \\
\hline Veronica sublobata M. A. Fisch. & 1 & $\mathrm{~T} 1$ & Ap1 & 15 & $\mathrm{~S}$ \\
\hline Vinca minor L. & 1 culA & $\mathrm{C} 1$ & Er2 & 17 & $\mathrm{~S}$ \\
\hline Viola cyanea Čelak. & $+\operatorname{culA}$ & H1 & $\mathrm{Kn}$ & 3 & $\mathrm{~S}$ \\
\hline Viola odorata L. & $+\operatorname{culA}$ & H1 & $\mathrm{Kn}$ & 3 & S \\
\hline Viola $\times$ wittrockiana Hort. & + culB & T0 & Er2 & 14 & $S$ \\
\hline Waldsteinia ternata (Stephan) Fritsch & 1 culB & $\mathrm{H} 1$ & Er1 & 17 & \\
\hline
\end{tabular}

Explanations:

Groundcover plants: old and recent.

FC (Frequency classes): R - 1-2 plants; + - several plants; 1 - very rare (covering $1-5 \%$ of cemetery area excluding graves); 2 - rare $(5-25 \%) ; 3$ - moderately frequent $(25-50 \%) ; 4-$ common $(50-75 \%) ; 5$ - very frequent $(75-100 \%)$; cul - cultivated, introduced long ago (A) or recently (B), i.e. in the last 10 years or so.

LF (Life-forms): F1 - megaphanerophytes; F2 - nanophanerophytes; C1 - woody chamaephytes; C2 - non-woody chamaephytes; G1 - wintering geophytes; G0 - non-wintering geophytes; H1 - wintering hemicryptophytes; H0 - non-wintering hemicryptophytes; $\mathrm{T} 1$ - annual 
therophytes; T2 - biennial therophytes; T0 - non-wintering therophytes; li - lianas; pp - parasites; ppep - hemiparasites.

GH (Geographic-historical status): Sp1 - spontaneous spontaneophytes; Sp2 - planted spontaneophytes; Ap1 - spontaneous apophytes; Ap2 - planted apophytes; Arch - archaeophytes; Ken - kenophytes; Er1 - non-spreading ergasiophytes; Er2 - ergasiophytes spreading within the cemetery.

SE (socioecological groups): 1 - fertile broad-leaved forests and shrub communities (Fagetalia, Prunetalia); 2 - acidophilous or xerothermic oak forests, mixed coniferous forests and their substitute shrub, herb or grassland communities (Quercion robori-petraeae, Quercion petraeae, Epilobion, Nardetalia); 3 - nitrophilous shrub or herb communities (Sambuco-Salicion, Alliarion); 4 - xerothermic herb or grassland communities (Trifolio-Geranietea, Festuco-Brometea); 5 - pine forests or sandy grasslands (Dicrano-Pinion, Sedo-Scleranthetea, Corynephoretea); 6 - swamp alder forests, woodless fens, bogs and intermediate mires, and riparian forests and thickets, reeds and aquatic vegetation (Alnion, Magnocaricion, Caricetalia fuscae, Sphagnion fusci, Salicion, Phragmition, Glycerio-Sparganion, Potamogetonetea, Lemnetea, Utricularietea); 7 - humid meadows and tall herb communities (Molinietalia); 8 - fresh and moderately humid meadows (Arrhenatheretalia); 9 - nitrophilous floodplains and treaded communities (Plantaginetea); 10 therophyte communities on wet and humid sites (Bidentetea, Nanocyperion); 11 - mesophilous communities of tall perennials (Arction); 12 - xerothermic, perennial ruderal communities (Onopordon); 13 - short-term, pioneer ruderal communities (Sisymbrion, Eragrostion); 14 - weed communities of gardens and root crop fields (Polygono-Chenopodietalia, Poo-Oxalidetum); 15 weed communities of cereal fields (Aperetalia); 16 - epilithic communities (Asplenietea); 19 species with undefined phytosociological affiliation.

$\mathrm{S}-$ species with a symbolic meaning.

$\mathrm{C} \mathrm{Cz}-$ species with a partial protection.

The tree layer consisted of 15 taxa, the shrub layer of 26, and the herb layer of 99 taxa. It must be emphasized that the tree layer included Fraxinus excelsior 'Pendula', which symbolizes the hair of a girl weeping at her friend's grave. In the shrub layer, only three species occurred spontaneously: Fraxinus pennsylvanica, R. pseudoacacia, and Sambucus nigra. Abundantly fruiting Buxus sempervirens reached $2 \mathrm{~m}$ in height. In the herb layer, 51 species were native to the study area, but 13 of them were planted, e.g. Aegopodium podagraria, Allium vineale, Anemone ranunculoides, Aquilegia vulgaris, Campanula rapunculoides, Convallaria majalis, Dryopteris filix-mas, Ficaria verna, Gagea lutea, G. pratensis, Galanthus nivalis, Lysimachia nummularia, and Taxus baccata. Among alien herbaceous species, 31 were planted: Alchemilla mollis, Allium scorodoprasum, Bergenia cordifolia, Cotoneaster horizontalis, C. suecicus, Eranthis hyemalis, Fragaria indica, Gagea arvensis, Galeobdolon argentatum, Geranium macrorrhizum, G. platypetalum, Hedera helix, Hemerocallis fulva, Ligularia dentata, Matteucia struthiopteris, Muscari botryoides, Ornithogalum nutans, O. umbellatum, Pachysandra terminalis, Reynoutria japonica, Scilla sibirica, Sedum specta- 
bile, Tradescantia $\times$ andersoniana, Tulipa gesneriana, T. sylvestris, Vinca minor, Viola cyanea, $V$. odorata, $V$. ×wittrockiana, and Waldsteinia ternata.

In the tree layer, the major species (with the highest degree of cover) were: A. platanoides, A. hippocastanum, F. excelsior, Quercus robur, and T. cordata (Tab. 1). The shrub layer was poorly developed. Shrubs were the most numerous in the meadow part, with no monuments, where the highest degree of cover was recorded for Cornus stolonifera. In the part with graves, the most abundant shrub species was Symphoricarpos albus. In the herb layer, the major species were D. filix-mas, F. verna, Gagea lutea, H. helix, Lolium perenne, Poa pratensis, S. sibirica, Taraxacum officinale, and T. sylvestris. All the major herb species except T. officinale were planted in the part with graves or sown in the meadow.

In the flora of the Cemetery of the Meritorious, species in the second frequency class $(+)$ were the most numerous (over $52 \%$ of the total number of species). Species in the third frequency class (1) were less numerous (over 23\%). Species in the first class (R) and fourth class (2) accounted for about $10 \%$ each. The fifth frequency class (3) was represented by only two species: A. platanoides in the tree layer and H. helix in the herb layer (Tab. 2).

Table 2. Percentage contributions of species of individual frequency classes to the total flora in the Cemetery of the Meritorious in Poznan

\begin{tabular}{|c|l|c|c|}
\hline \multicolumn{2}{|c|}{ Frequency class (FC) } & Number of species & \% of total flora \\
\hline$R$ & $1-2$ specimens & 20 & 13.6 \\
\hline+ & several specimens & 76 & 51.7 \\
\hline 1 & $\begin{array}{l}\text { very rare species (covering 1-5\% } \\
\text { of cemetery area excluding graves) }\end{array}$ & 36 & 24.5 \\
\hline 2 & rare (5-25\%) & 13 & 8.8 \\
\hline 3 & moderately frequent (25-50\%) & 2 & 1.4 \\
\hline 4 & frequent (50-75\%) & 0 & 0 \\
\hline 5 & very frequent (75-100\%) & 0 & 0 \\
\hline
\end{tabular}

Among geographical-historical groups, native species (apophytes) account for over $43 \%$ of the flora of the study area (Tab. 3). Alien species include archaeophytes (more than $8 \%$ of the flora), kenophytes (14.3\%), and ergasiophytes (35.4\%). The most noteworthy archaeophyte is Gagea arvensis. Kenophytes are represented by e.g. A. hippocastanum, Ailanthus altissima, A. scorodoprasum, Conyza canadensis, Geranium molle, H. helix, O. umbellatum, Oxalis dillenii, $O$. fontana, V. cyanea, and V. odorata. Ergasiophytes are an important group in old cemeteries, here comprising e.g. A. mollis, B. cordifolia, E. hyemalis, Fragaria indica, G. argentatum, G. macrorrhizum, G. platypetalum, H. fulva, M. struthiopteris, $O$. nutans, $P$. terminalis, $R$. japonica, $S$. sibirica, $S$. spectabile, $T$. $\times$ andersoniana, T. gesneriana, $T$. sylvestris, $V$. minor, and $V$. $\times$ wittrockiana. 
Pobrane z czasopisma Annales C - Biologia http://biologia.annales.umcs.pl

Data: 26/04/2023 14:01:33

68

ANETA CZARNA

Table 3. Percentage contributions of geographical-historical groups to the total flora in the Cemetery of the Meritorious in Poznan

\begin{tabular}{|c|l|c|c|}
\hline \multicolumn{2}{|c|}{ Geographic-historical status (GH) } & Number of species & \% of total flora \\
\hline Sp1 & spontaneous spontaneophytes & 2 & 1.4 \\
\hline Sp2 & planted spontaneophytes & 3 & 2.0 \\
\hline Ap1 & spontaneous apophytes & 39 & 26.5 \\
\hline Ap2 & planted apophytes & 18 & 12.2 \\
\hline Ar & archaeophytes & 12 & 8.2 \\
\hline Kn & kenophytes & 21 & 14.3 \\
\hline Er1 & non-spreading ergasiophytes & 47 & 32.0 \\
\hline Er2 & ergasiophytes spreading within cemetery & 5 & 3.4 \\
\hline
\end{tabular}

Among socioecological groups (Tab. 4), the major group are the taxa with undefined phytosociological affiliation (group 17=39.5\%). Among the species attributed to a defined group, a major role is played by species of fertile deciduous forests and shrub communities (group 1=13\%), weeds of gardens and root crop fields (group 14=10.3\%), plants of nitrophilous shrub communities and forest edges (group $3=9.5 \%$ ), and of fresh and moderately moist meadows (group $8=6.1 \%$ ). Contributions of other groups vary from $1.4 \%$ to $4.8 \%$.

Table 4. Percentage contributions of socioecological groups to the total flora in the Cemetery of the Meritorious in Poznań

\begin{tabular}{|c|l|c|c|}
\hline \multicolumn{2}{|c|}{ Socioecological groups (SE) } & $\begin{array}{c}\text { Number } \\
\text { of species }\end{array}$ & \% of total flora \\
\hline 1 & $\begin{array}{l}\text { Fertile broad-leaved forests and shrub communities } \\
\text { (Fagetalia, Prunetalia) }\end{array}$ & 19 & 12.9 \\
\hline 2 & $\begin{array}{l}\text { Acidophilous or xerothermic oak forests, mixed coniferous } \\
\text { forests, and their substitute shrub, herb or grassland } \\
\text { communities (Quercion robori-petraeae, Quercion } \\
\text { petraeae, Epilobion, Nardetalia) }\end{array}$ & 3 & 2.0 \\
\hline 3 & $\begin{array}{l}\text { Nitrophilous shrub or herb communities } \\
\text { (Sambuco-Salicion, Alliarion) }\end{array}$ & 4 & 2.7 \\
\hline 4 & $\begin{array}{l}\text { Xerothermic herb or grassland communities } \\
\text { (Trifolio-Geranietea, Festuco-Brometea) }\end{array}$ & 2.0 \\
\hline 5 & $\begin{array}{l}\text { Pine forests or sandy grasslands (Dicrano-Pinion, } \\
\text { Sedo-Scleranthetea, Corynephoretea) }\end{array}$ & 2 & 1.4 \\
\hline 6 & $\begin{array}{l}\text { Swamp alder forests, woodless fens, bogs, and } \\
\text { intermediate mires and riparian forests and thickets, } \\
\text { reeds, and aquatic vegetation (Alnion, Magnocaricion, } \\
\text { Caricetalia fuscae, Sphagnion fusci, Salicion, } \\
\text { Phragmition, Glycerio-Sparganion, Potamogetonetea, } \\
\text { Lemnetea, Utricularietea) }\end{array}$ & 2.7 \\
\hline
\end{tabular}


Pobrane z czasopisma Annales C - Biologia http://biologia.annales.umcs.pl

Data: 26/04/2023 14:01:33

VASCULAR PLANTS IN THE CEMETERY OF THE MERITORIOUS IN POZNAŃ (POLAND)

Tab. 4

\begin{tabular}{|c|c|c|c|}
\hline \multicolumn{2}{|r|}{ Socioecological groups (SE) } & \multirow{2}{*}{$\begin{array}{c}\begin{array}{c}\text { Number } \\
\text { of species }\end{array} \\
1 \\
\end{array}$} & \multirow{2}{*}{\begin{tabular}{|c|}
$\%$ of total flora \\
0.7 \\
\end{tabular}} \\
\hline 7 & Humid meadows and tall herb communities (Molinietalia) & & \\
\hline 8 & Fresh and moderately humid meadows (Arrhenatheretalia) & 9 & 6.1 \\
\hline 9 & $\begin{array}{l}\text { Nitrophilous floodplains and treaded communities } \\
\text { (Plantaginetea) }\end{array}$ & 5 & 3.4 \\
\hline 10 & $\begin{array}{l}\text { Therophyte communities on wet and humid sites } \\
\text { (Bidentetea, Nanocyperion) }\end{array}$ & 1 & 0.7 \\
\hline 11 & Mesophilous communities of tall perennials (Arction) & 2 & 1.4 \\
\hline 12 & Xerothermic, perennial ruderal communities (Onopordion) & 5 & 3.4 \\
\hline 13 & $\begin{array}{l}\text { Short-term, pioneer ruderal communities } \\
\text { (Sisymbrion, Eragrostion) }\end{array}$ & 3 & 2.0 \\
\hline 14 & $\begin{array}{l}\text { Weed communities of gardens and root crop fields } \\
\text { (Polygono-Chenopodietalia, Poo-Oxalidetum) }\end{array}$ & 15 & 10.3 \\
\hline 15 & Weed communities of cereal fields (Aperetalia) & 3 & 2.0 \\
\hline 16 & Epilithic communities (Asplenietea) & 0 & 0.0 \\
\hline 17 & Species with undefined phytosociological affiliation & 58 & 39.5 \\
\hline
\end{tabular}

Among Raunkiaer's life forms (Tab. 5) in the study area, hemicryptophytes are the most diverse, represented by 45 species $(30.6 \%)$. Contributions of megaphanerophytes, nanophanerophytes, and annual therophytes exceed $15 \%$ each, while geophytes account for more than $12 \%$.

Table 5. Percentage contributions of plant life forms to the total flora in the Cemetery of the Meritorious in Poznań

\begin{tabular}{|c|l|c|c|}
\hline \multicolumn{2}{|c|}{ Life forms (LF) } & Number of species & \% of total flora \\
\hline F1 & megaphanerophytes & 25 & 17.0 \\
\hline F2 & nanophanerophytes & 22 & 15.0 \\
\hline C1 & woody chamaephytes & 5 & 3.4 \\
\hline C2 & non-woody chamaephytes & 1 & 0.7 \\
\hline H1 & wintering hemicryptophytes & 46 & 31.2 \\
\hline G1 & wintering geophytes & 19 & 12.9 \\
\hline T0 & non-wintering therophytes & 1 & 0.7 \\
\hline T1 & annual therophytes & 26 & 17.7 \\
\hline T2 & biennial therophytes & 2 & 1.4 \\
\hline
\end{tabular}

Special attention was paid to groundcover plants, which decorate graves and their vicinity. These include perennial herbs, dwarf shrubs or low shrubs, forming dense patches, either natural or introduced intentionally, protecting the soil from expansion of weeds, erosion or degradation (14). Among them, 21 taxa were introduced long ago (A. podagraria, A. scorodoprasum, $A$. ranunculoides, $C$. ma- 
jalis, F. verna, G. lutea, G. pratensis, H. helix, H. fulva, Lysimachia nummularia, M. struthiopteris, O. nutans, O. umbellatum, $S$. sibirica, $V$. minor, V. cyanea, and $V$. odorata) and 15 recently (A. mollis, C. horyzontalis, C. suecicus, D. filix-mas, E. hyemalis, F. indica, G. argentatum, G. macrorrhizum, G. platypetalum, P. terminalis, R. japonica, S. spectabile, T. baccata, T. ×andersoniana, and V. ternata).

The genus Gagea was represented by three species: G. arvensis, G. lutea, and G. pratensis. The first two species were certainly introduced intentionally, while Gagea pratensis probably accidentally, accompanying other plant species introduced into this cemetery when it was created.

Plants in cemeteries were attributed symbolic meanings related to religion already in the Middle Ages. First of all, they were supposed to be associated with concepts of the future paradise. Many of the plants cultivated in cemeteries were worshipped according to folk beliefs in the given region. Among the plants with symbolic meanings, 13 species are in the trees, 16 in the shrubs, and 30 in the herbaceous plants (Tab. 1). Particularly noteworthy are $V$. minor and H. helix, which symbolize immortality and paradise, but also friendship and faithfulness. Trifoliate leaves, like those of $F$. indica (symbol of noble modesty and humility) and clovers (Trifolium spp.), symbolized the Holy Trinity. The species flowering in spring included: 1 . A ranunculoides, withering quickly and associated with disease and death, also symbolizing the shed blood of saints; 2. C. majalis, symbolizing youth, chastity, and salvation; 3 . Galanthus nivalis, a symbol of spring and Christian hope; 4. Viola odorata, because of its subtle scent and dark purple flowers regarded as a symbol of modesty and humility; 5. Myosotis sp., a sign of eternal sleep and memory of the dead; 6 . pansy $V$. × wittrockiana, planted every year, reminding about the deceased, being also a sign of the Holy Trinity. In the meadow part of the cemetery, Bellis perennis is very common. It grows there spontaneously and symbolizes modesty, innocence, immorality, and eternal life. Spontaneous species with symbolic meanings include also Veronica sublobata, Geum urbanum, and Chelidonium majus; their symbolism is similar, denoting salvation and redemption from spiritual blindness. Particular power, symbolizing resurrection and restoration of vitality, was attributed to trees, which "restore life" every year. They are represented in the study area by 10 species. Buxus sempervirens was believed to deter evil spirits and regarded as a tree of life and death, as well as constancy and power. The presence of $R$. pseudoacacia was linked with old beliefs and fear of penetration of the dead from the cemetery to the living world. The space under Sambucus nigra was magical, people believed that evil spirits found shelter there. After washing the dead body, the water was poured under this bush, to protect other members of the family against early death. Roses (Rosa spp.) have many symbolic meanings, depending on flower colour and the presence/absence of prickles. Generally, however, roses are associated with Mary 
and Christ's blood, transition, love, and death. The most common species in the cemetery is A. platanoides, which was believed to deter devils waiting for the souls of the dead. Q. robur, according to many beliefs, was created as the first tree. In the imagery, the oak was gigantic, a sign of immortality, power, and might. Tilia sp. guaranteed peaceful eternal sleep. The currently very common cemetery species Thuja occidentalis is a sign of sorrow, death, and mourning.

Currently the symbolism of cemetery plants is of little significance, as they are cultivated primarily because of their ornamental value. Recently introduced species include $A$. mollis (decorative habit and leaves), Cotoneaster horizontalis and C. suecicus (decorative habit and fruits), E. hyemalis (decorative habit and flowers), F. indica (decorative flowers, leaves, and habit), D. filix-mas (decorative habit), Geranium macrorrhizum (decorative habit and flowers), G. platypetalum (decorative habit and flowers), P. terminalis (decorative habit and leaves), $R$. japonica (decorative habit and leaves), S. spectabile (decorative habit and flowers), $T$. $\times$ andersoniana (decorative habit), and $W$. ternata (decorative habit and leaves).

\section{DISCUSSION}

In the Cemetery of the Meritorious in Poznań, 140 taxa of vascular plants were recorded. The trees consisted of 15 taxa, the shrubs of 26, and the herbaceous plants of 99 . As many as 84 of them were planted, mostly in the herb layer: 14 native species and 31 alien species.

Comparable numbers of plant species were found in some other old cemeteries, both in Poland, e.g. 144 in the New Cemetery in Zakopane (4) and in other countries, e.g. recently 143 in Timișoara in Bulgaria (16) and nearly 30 years ago in Australia: 136 in Rossmore and 148 in an Anglican cemetery in Campbelltown (14). However, in many other cemeteries the numbers of recorded plant species were much lower or higher, e.g. 51 in Greendale and 164 in Liverpool in Australia (14) and 363 wild-growing plant species in the Jewish cemetery in Berlin (1).

The assumption that apophytes are not numerous was not confirmed. They ranked first among geographic-historical groups ( $41 \%$ of total flora). Their high contribution resulted from introduction of over $15 \%$ species to cultivation, as well as from the forest-like vegetation and magnificent, about 200-year-old trees in a large part of the cemetery.

140 species of vascular plants were found in the Meritorious Cemetery, and 255 species of vascular flora were found in cemeteries in Poznan (3). On the other hand, the dendropflora of the selected Lubelszczyzna cemeteries has 114 species (8). Among the plants with symbolic meanings, 13 species were in the trees, 16 in the shrubs, and 30 in the herbaceous plants. The presented results show that $62.5 \%$ of the shrubs found in the Cemetery of the Meritorious in Poznan have symbolic meanings. In contrast, in cemeteries of the Lublin region, symbolic shrubs 
account for 50\% (8), while in the Citadel of Poznań, only 28.1\% (3). Contributions of symbolic plants were higher also in the other layers of vegetation: $86 \%$ of trees in the Cemetery of the Meritorious, compared to $41.4 \%$ in the Citadel (3) and $30.2 \%$ of herbs in the study area, compared to $17.9 \%$ in the Citadel (3). Similarly, groundcover plants accounted for $24 \%$ in the Cemetery of the Meritorious, while in the Citadel of Poznań, for 9.5\% (3).

In total, groundcover plants were represented by 35 species (24\%), 21 of them introduced long ago, and 15 recently (during the last 10 years or so). Ground - cover plants are also popular in other cemeteries.

\section{REFERENCES}

1. Buchholz S., Blick T.H., Hannig K., Kowarik I., Lemke A., Otte I., Scharon J., Schönhofer A., Teige T., von der Lippe M., Seitz B., 2016. Biological richness of a large urban cemetery in Berlin. Results of a multi-taxon approach. Biodivers. Data J. (4): e7057.

2. Buczacki S., 1997. Rośliny w zacienionym ogrodzie. Elipsa.

3. Czarna A., 2016. Vascular plant flora in the Cytadela cemeteries in Poznań (Poland). Acta Agrobot. 69(4): 1-17.

4. Czarna A., Piskorz R., 2005. Vascular flora of cemeteries in the town of Zakopane in the Tatra Mountains. Rocz. AR Pozn. 373, ser. Bot.-Stec. 9: 47-58.

5. Ellis B. W. 2008. Rośliny okrywowe. Byliny, pnącza i krzewy zamiast trawnika. Klub dla Ciebie.

6. Erhardt W., Götz E., Bödeker N., Seyboid S. 2008. Zander. Handwörterbuch der Pflanzennamen, Ulmer, pp. 983.

7. Gawryś W. 2008. Słownik Roślin Zielnych. Łacińsko-Polski. Officina Botanica, Kraków.

8. Karczmarz K., Trzaskowska E., 2013. Analiza dendroflory założeń cmentarnych w krajobrazie miasta i wsi Lubelszczyzny. Teka Kom. Arch. Urb. Stud. Krajobr. - OL PAN, 9(4): 7-20.

9. Knaflewska J., 2006. Zieleń na cmentarzach. Zieleń Miejska, 9: 13.

10. Kobielus S. 2006. Florarium christianum. Symbolika roślin - chrześcijańska starożytność i średniowiecze. Tyniec, Wydawnictwo Benedyktynów. Kraków, pp. 255.

11. Kopaliński W., 1985. Słownik mitów i tradycji kultury. Państwowy Instytut Wydawniczy, Warszawa.

12. Linette R., Matysiak J., 2013. Cmentarze i Krypta Zasłużonych w Poznaniu. Wydawnictwo Miejskie Posnania.

13. Łukaszewicz A., 2003. Rośliny okrywowe. Państwowe Wydawnictwo Rolnicze i Leśne.

14. Mc Barron E. J., Benson D. H., Doherty M. D., 1988. The botany of old cemeteries. Cunninghamis, 2(1): 97-105.

15. Mirek Z., Piękoś-Mirkowa H., Zając A., Zając M., 2002. Flowering plants and pteridophytes of Poland. A checklist. Krytyczna lista roślin naczyniowych Polski. Biodiversity of Poland, vol. 1. W. Szafer Institute of Botany, Polish Academy of Science, Kraków.

16. Otves C., Arsene G.-G., Neacşu A., 2016. Species diversity of the plants found in the RomanCatholic and Orthodox cemeteries (from the Mehala Neighbourhood) and the heroes cemetery from Timisoara. Research Journal of Agricultural Science, vol. (48(2): 82-92).

17. Richter G., 1993. Kryteria planowania zieleni na cmentarzach. [In:] Struktura cmentarna, ICOMOS. O. Cerner, I. Juszkiewicz (eds). Polish National Committee, Museum of Art Wrocław, Wrocław. 
Pobrane z czasopisma Annales C - Biologia http://biologia.annales.umcs.pl

Data: 26/04/2023 14:01:33

18. Rutkowski L., 1998. Klucz do oznaczania roślin naczyniowych Polski niżowej. Wydawnictwo Naukowe PWN, Warszawa.

19. Zarzycki K., Trzcińska-Tacik H., Różańska W., Szeląg Z., Wołek J., Korzeniak U., 2002. Ecological indicator of vascular plants of Poland. W. Szafer Institute of Botany, Polish Academy of Science, Kraków.

20. Ziółkowska M., 1988. Gawędy o drzewach. Ludowa Spółdzielnia Wydawnicza, Warszawa.

\section{ACKNOWLEDGEMENTS}

I am grateful to Professor Jerzy Zieliński of the Institute of Dendrology in Kórnik, Poland, for verification and identification of the collected herbarium specimens of trees and shrubs. The study was supported by the National Science Centre in Kraków, Poland (grant no. NCN NN304204937). The manuscript was partly translated into English by Sylwia Ufnalska. 